\title{
REVISIÓN DE TRABAJOS CIENTÍFICOS \\ Polimorfismos genéticos y su asociación con proteínas alteradas en individuos ecuatorianos afectos con cáncer
}

\author{
Andrés López-Cortés, María José Muñoz, César Paz-y-Miño
}

Instituto de Investigaciones Biomédicas. Facultad de Ciencias de la Salud. Universidad de las Américas. Av. de los Granados E12-41 y Colimes. Quito, Ecuador.

Código postal: 1712842 .

cpazymino@udla.edu.ec

Recibido: 16, 04, 2011; aprobado: 30, 06, 2011

\begin{abstract}
RESUMEN.- El cáncer es un grupo de enfermedades causadas por factores externos e internos, y caracterizadas por el crecimiento y la dispersión de células anormales. El cáncer de piel ha incrementado el número de casos en los últimos años mientras que el cáncer pulmonar presenta el mayor índice de mortalidad en países industrializados. En Quito, los tipos más frecuentes de cáncer en hombres son próstata, piel y estómago mientras que en mujeres son piel, mama y cérvix invasor. La determinación de las distribuciones genotípicas y las frecuencias alélicas de polimorfismos en los genes SRD5A2 en cáncer de próstata, GPX-1 y MnSOD en cáncer de vejiga y EGFR en cáncer pulmonar, se realizó a través de la técnica bialélica PCR-RFLP, y sus datos fueron analizados mediante chi-cuadrado $\left(\chi^{2}\right)$ y odds ratio (OR). Como resultados, las variantes genéticas V89L (SRD5A2) (OR $3.7, p<0.001$ ), P198L (GPX-1) (OR 3.8; $p<0.001$ ) y L858R (EGFR) (OR 5.9; $p<$ 0.001 ), presentaron diferencias significativas y son factores de riesgo para el desarrollo del cáncer. En conclusión, la predisposición de una persona para desarrollar cáncer dependerá de la exposición a agentes cancerígenos y de su perfil genético, el cual influirá en la síntesis proteica y en el mal funcionamiento de los mecanismos celulares.
\end{abstract}

PALABRAS CLAVE: Ca próstata, Ca vejiga, Ca pulmón, EGFR, GPX-1, MnSOD, SRD5A2

ABSTRACT.- Cancer is a group of diseases caused by external and internal factors, and characterized by uncontrolled growth and spread of abnormal cells. Skin 
cancer cases have drastically increased over the years while lung cancer has the highest mortality index in developed countries. In Quito the most frequent types of cancer in men are prostate, skin, and stomach, while in women are skin, breast, and cervix invasive. The determination of the genotypic distribution and the allele frequencies of polymorphisms in the SRD5A2 genes in prostate cancer, GPX-1 and MnSOD in bladder cancer, and EGFR in lung cancer were performed through the biallelic marker PCR-RFLP, and data were analyzed by chi-square $\left(\chi^{2}\right)$ and odds ratio tests (OR). As a result, the genetic variants V89L (SRD5A2) (OR 3.7; $p<$ 0.001), P198L (GPX-1) (OR 3.8; $p<0.001$ ) and L858R (EGFR) (OR 5.9; $p<$ 0.001 ) have been detected as risk factors for prostate, bladder, and lung cancer development, respectively. In conclusion, a person's predisposition to develop cancer is not only influenced by exposure to carcenogenic agents but also it will depend on genetic profile, which will directly influence in protein synthesis, as well as the malfunction of cells' mechanisms.

KEYWORDS: Prostate Ca, Bladder Ca, Lung Ca, EGFR, GPX-1, MnSOD, SRD5A2

\section{INTRODUCCIÓN}

El cáncer es el resultado de dos procesos sucesivos: el aumento de la proliferación de células tumorales y la posterior adquisición de la capacidad invasiva, proceso denominado metástasis (1). Debido a la acumulación de alteraciones en los genes durante el período de crecimiento, las células tumorales activan cascadas genéticas que desencadenan en el aumento de la proliferación y diferenciación celular, así estas células son seleccionadas positivamente debido a su mayor adaptación a nivel evolutivo (2). Existen alrededor de 200 tipos de células que al alterarse genéticamente pueden formar tumores y desarrollar cáncer, y se las clasifica de acuerdo a su origen: los tumores generados por célu- las epiteliales son carcinomas, por células del tejido conectivo son sarcomas, por células sanguíneas son leucemias, linfomas y mielomas, y por células del sistema nervioso son gliomas (3).

Existen cuatro tipos de genes relacionados con el cáncer: los oncogenes mutan y originan proteínas con funciones alteradas que estimulan el crecimiento o invasividad celular; los genes supresores de tumores controlan el ciclo de división celular, evitando el crecimiento excesivo y la formación de tumores; los genes reparadores del ADN son responsables de reparar los errores producidos en la incorporación de nucleótidos durante la replicación del material genético; y los genes reguladores de apoptosis controlan la muerte celular programada (3). 
El cáncer puede ser causado por factores externos como el tabaquismo, la exposición a químicos, hidrocarburos, pesticidas o radiación ionizante, y por factores internos como mutaciones heredadas, hormonas, condiciones inmunológicas y mutaciones que ocurren como consecuencias metabólicas $(4,5,6)$.

Con relación a la incidencia de cáncer a nivel mundial, en Estados Unidos, los carcinomas de próstata, pulmón y colon correspondieron a los primeros lugares con relación a los nuevos casos estimados en hombres, y los carcinomas de mama, pulmón y colon con relación a los nuevos casos estimados en mujeres en el año 2010 (1). Con respecto al índice de mortalidad tanto en hombres como en mujeres, el cáncer de pulmón y bronquios ocupó el primer lugar en el año 2010 en Estados Unidos (1). El Ecuador se ubica en el quincuagésimo segundo puesto en relación a las tasas más altas de incidencia de cáncer tanto en hombres como en mujeres a nivel mundial. Los tipos más frecuentes de cáncer en hombres en Quito entre los años 2003 - 2005 fueron: próstata, piel, estómago, sistema hematopoyético, ganglios linfáticos $\mathrm{y}$ pulmón; mientras que en mujeres fueron: piel, mama, cérvix invasor, estómago, tiroides y sistema hematopoyético (7).

Con respecto al cáncer de próstata, se ha reportado que la población afroamericana presenta la tasa más alta de incidencia, seguido de los caucásicos y los asiáticos $(8,9)$. A partir de 1990 se aplicó en el diagnóstico del cáncer de prós- tata la determinación de los niveles de antígeno prostático específico (PSA) en las glándulas prostáticas (7). La función del gen SRD5A2 es catalizar, mediante la enzima esteroide $5 \alpha$-reductasa de tipo II, la reducción irreversible de la testosterona, en el andrógeno más activo, la $5 \alpha$-dihidrotestosterona (DHT). Una vez sintetizada, la DHT se une al receptor de andrógenos y es transportada al núcleo. Dentro del núcleo, este complejo se une a genes blanco e inicia la transcripción de factores de crecimiento para controlar la proliferación, diferenciación y regulación del ciclo celular $(10,11,12$, 13). Debido a que la principal función de SRD5A2 se encuentra en la vía metabólica del andrógeno, se ha propuesto que sus variantes polimórficas (V89L A497T) podrían ser factores de riesgo para la progresión y desarrollo del cáncer de próstata (14).

En países industrializados, el cáncer de vejiga es el cuarto tipo de carcinoma más frecuente diagnosticado en hombres y noveno en mujeres (15). Alrededor de 357.000 nuevos casos son reportados anualmente siendo este cáncer el más frecuente desarrollado en el tracto urinario (16). En Ecuador, el carcinoma de vejiga representa la decimonovena y veinteava causa de muerte en hombres y mujeres, respectivamente (17). Ciertas enzimas que no neutralizan ni degradan de forma adecuada los radicales oxidativos, los acumulan destruyendo las células epiteliales de la vejiga, causando 
alto riesgo de adquirir cáncer (18). El estrés oxidativo es caracterizado por el incremento de especies oxígeno y nitrógeno reactivas y por la reducción de la actividad de enzimas antioxidantes, las cuales causan disfunciones metabólicas y daño en las macromoléculas biológicas. Las enzimas detoxificadoras, inactivan compuestos y aniones altamente peligrosos para la célula. Las defensas contra especies oxígeno reactivas están conformadas por glutatión peroxidasa (GPX-1), catalasa (CAT) y superóxido dismutasa (SOD) (18). Se ha reportado que la presencia de los polimorfismos P198L (GPX-1) e I58T (MnSOD) se asocian con el incremento en el riesgo de adquirir cáncer de vejiga $(18,19,20)$.

Según la Sociedad Americana contra el Cáncer, el carcinoma pulmonar representa la principal causa de muerte y es la neoplasia con mayor incidencia en países industrializados (1). En Ecuador, el cáncer de pulmón es la quinta neoplasia con mayor incidencia en hombres (7). El gen EGFR (receptor del factor de crecimiento epidermal) codifica una glicoproteína integral de membrana que presenta actividad tirosina quinasa (21). La fosforilación del dominio quinasa de la proteína inicia una cascada de señales intracelulares con el fin de regular la proliferación, diferenciación celular y angiogénesis (22). La presencia de las mutaciones somáticas L858R y G719S, constituyen una de las mayores anomalías genéticas presentes en el gen EGFR, debido a que se correlacionan con la presencia de factores oncogénicos y mecanismos de apoptosis celular $(23,24)$.

El estudio de los polimorfismos V89L y A497T (SRD5A2) en cáncer de próstata, P198L (GPX-1) y I58T (MnS$\mathrm{OD})$ en cáncer de vejiga, y de las mutaciones L858R y G719S (EGFR) en cáncer de pulmón, tiene como objetivo caracterizar genéticamente a la población ecuatoriana y conocer más profundamente el funcionamiento de los genes envueltos en el origen, progresión y evolución de los tumores.

\section{MATERIALES Y MÉTODOS}

Muestras biológicas.- Para realizar el análisis genético a la población ecuatoriana se obtuvieron 291 muestras de tumores frescos y embebidos en parafina de pacientes diagnosticados con carcinoma de próstata, vejiga y pulmón. La obtención de las muestras tumorales se realizó a través del Departamento de Urología del Hospital Carlos Andrade Marín del Instituto Ecuatoriano de Seguridad Social (IESS) y del Departamento de Patología del Hospital Oncológico Solón Espinoza Ayala de la Sociedad de Lucha Contra el Cáncer (SOLCA). En cuanto a individuos control, se obtuvieron 373 muestras de sangre periférica de individuos sanos sin antecedentes de tabaquismo, ni exposición a agentes carcinógenos. Cada uno de ellos lleno una encuesta biomédica y su respectivo consentimiento informado. 
Análisis de polimorfismos genéticos.- El ADN de los individuos afectos con cáncer y de individuos sanos, almacenado en el banco de ácidos nucleicos del Instituto de Investigaciones Biomédicas, se lo extrajo mediante el uso del kit DNA Genomic Wizard Purification (Promega, Madison WI, USA). Para obtener el genotipo de cada uno de los individuos sanos y afectos, se procedió a utilizar la reacción en cadena de la polimerasa (PCR). La PCR es una técnica de Biología Molecular descrita por Kary Mullis en 1986 (25), esta reacción es una forma de clonación in vitro que es utilizada en la amplificación de pequeñas cantidades de $\mathrm{ADN}$ a partir de la activación de la enzima ADN polimerasa proveniente de una bacteria termófila (Thermus acuaticus) (26).

La primera generación de marcadores moleculares de ADN fueron los fragmentos de restricción de longitud polimórfica (RFLP). La técnica PCR-RFLP es un marcador genético bi-alélico que se basa en la presencia o ausencia de una secuencia. El producto de PCR es incubado con la enzima de restricción específica y luego es testado para observar si el fragmento de ADN ha sido cortado (27). Dentro de la semejanza entre los individuos de una misma especie, existen variaciones individuales en la secuencia de la información genética. Los polimorfismos consisten en variaciones en la secuencia del ADN. Las enzimas de restricción ponen de manifiesto la presencia o ausencia de los polimorfismos de nucleótido simple (SNP's), ya que un cambio en la secuencia del ADN puede crear o destruir un lugar de corte, dando lugar a una sobreexpresión o inhibición en las proteínas $(28,29)$.

Con respecto al cáncer de próstata, la amplificación del gen SRD5A2 se realizó en un volumen final de PCR de $100 \mu \mathrm{l}$, que contiene de 60 a $100 \mathrm{ng}$ de ADN templado, $3 \mathrm{mmol} / \mathrm{l}$ de $\mathrm{MgCl}_{2}, 10$ $\mu 1$ de PCR buffer 10X, $200 \mathrm{mM}$ de cada dideoxinucleótidos trifosfato (dNTP's), $0.001 \mathrm{mmol} / 1$ de DMSO (dimetil sulfóxido), $0.5 \mathrm{U}$ de Taq polimerasa, y 0.5 $\mu \mathrm{mol} / 1$ de primer sentido (FW) $5^{\prime}$ - TGG CCT TGT ACG TCG CGA AG -3' y del primer antisentido (RV) 5' - AGC AGG GCA GTG CGC TGC ACT -3' (30). Las muestras de PCR fueron amplificadas en un termociclador MJ Research PTC 200 (MJ-Research Inc., Watertown, MA). La reacción de PCR consistió en un ciclo de $94^{\circ} \mathrm{C}$ por $5 \min$ y $82^{\circ} \mathrm{C}$ por $1 \mathrm{~min}$ (denaturación inicial, seguido de 20 ciclos a $94^{\circ} \mathrm{C}$ por 1 min (denaturación), $68^{\circ} \mathrm{C}$ por $1 \mathrm{~min}$ con un decrecimiento de $0,5^{\circ} \mathrm{C}$ por ciclo (anillamiento), $72^{\circ} \mathrm{C}$ por 1 min (elongación), y 15 ciclos a $94^{\circ}$ $\mathrm{C}$ por $1 \mathrm{~min}$ (denaturación), $58^{\circ} \mathrm{C}$ por 1 min (anillamiento), $72^{\circ} \mathrm{C}$ por $1 \mathrm{~min}$ (denaturación) y por último $10 \mathrm{~min}$ a $72^{\circ} \mathrm{C}$ (elongación final) (30). Los amplicones se los migró por electroforesis en gel de agarosa al $2 \%$ con bromuro de etidio y se los observó en un transiluminador con luz ultravioleta (UV). La presencia 
de las variantes V89L y A49T fue determinada mediante la técnica PCR-RFLP, que consistió en la digestión de los amplicones durante 2 horas 30 minutos a $37^{\circ} \mathrm{C}$, usando $5 \mathrm{U}$ de las enzimas de restricción RsaI (corte romo; GT/AC) y MwoI (corte cohesivo; GCNNNNN/ NNGC), respectivamente. Los fragmentos resultantes se los observó mediante electroforesis, en un gel de agarosa al $3 \%$, y bajo luz UV (30).

Con relación al cáncer de vejiga, la amplificación de los genes GPX-1 (191 pb) y MnSOD (145 pb), se realizó en un volumen final de reacción de $25 \mu$ l. Cada muestra estuvo conformada por $10.85 \mu \mathrm{l}$ de agua Milli-Q, $2.5 \mu 1$ de PCR buffer 10x (200 mM Tris-HCl, pH 8.4, $500 \mathrm{mM} \mathrm{KCl}$ ) (Invitrogen, Carlsbad, CA), $0.8 \mu 1$ de $\mathrm{MgCl}_{2}$ a $2.5 \mathrm{mM}$, $2 \mu \mathrm{l}$ de dNTP's a $200 \mathrm{mM}, 2.5$ unidades de Taq polimerasa, $4 \mu \mathrm{l}$ de ADN a una concentración de $100 \mathrm{ng} / \mu 1$, y $2.5 \mu 1$ de primers FW y RV a $0.5 \mu \mathrm{M}$. Para el análisis del polimorfismo P198L se utilizaron los primers FW 5'- AAG GTG TTC CTC CCT CGT AGG T -3' y RV 5'- CTA CGC AGG TAC AGC CGC CGC T -3' (20). El paso de denaturación inicial duró 8 min a $94^{\circ} \mathrm{C}$, luego 36 ciclos de 1 min a $94^{\circ} \mathrm{C}$ (denaturación), 1 min a $60^{\circ} \mathrm{C}$ (anillamiento), 1 min a $72^{\circ} \mathrm{C}$ (elongación), y 9 min a $72^{\circ}$ $\mathrm{C}$ de elongación final. Para el análisis del polimorfismo I58T se utilizaron los primers FW 5' - ACT TCA GTG CAG GCT GAA CAGC -3'y RV 5'- CTG
GTC CCA TTA TCT AAT AGC TT-3'. Los pasos de la PCR fueron $10 \mathrm{~min}$ a $95^{\circ} \mathrm{C}$ (denaturación inicial), seguido de 35 ciclos de 30 seg. a $95^{\circ} \mathrm{C}$ (denaturación), $30 \mathrm{seg}$. a $55^{\circ} \mathrm{C}$ (anillamiento), 1 min a $72^{\circ} \mathrm{C}$ (elongación) y $10 \mathrm{~min}$ a $72^{\circ}$ C (elongación final) (20). Posteriormente, la presencia del polimorfismo P198L se determinó mediante el uso de ApaI (corte cohesivo; GGGCC/C), y el polimorfismo I58T mediante la enzima PvuI (corte cohesivo; CGAT/CG) (20).

Con respeto al cáncer de pulmón, la amplificación del gen EGFR se llevó a cabo en un volumen final de PCR de $25 \mu 1$ conteniendo $4 \mu \mathrm{l}$ de ADN a una concentración de $100 \mathrm{ng} / \mu 1,1,5 \mathrm{mM}$ de $\mathrm{MgCl}_{2}, 2,5 \mu \mathrm{l}$ de PCR buffer 10x, 200 $\mu \mathrm{M}$ de dNTP's, $2 \mathrm{U}$ de Taq polimerasa y $0,5 \mu \mathrm{M}$ de primers FW y RV. Para el análisis del polimorfismo G719S del exón 18 se utilizaron los primers FW 5' - TCC AAA TGA GCT GGC AAG TG -3' y RV 5' - TCC CAA ACA CTC AGT GAA ACA AA -3'. Los pasos de la PCR fueron $15 \mathrm{~min}$ a $95^{\circ} \mathrm{C}$ (denaturación inicial), seguido de 35 ciclos a $95^{\circ} \mathrm{C}$ por $20 \mathrm{seg}$ (denaturación), $57^{\circ}$ $\mathrm{C}$ por $20 \mathrm{seg}$ (anillamiento), $72^{\circ} \mathrm{C}$ por $30 \mathrm{seg}$ (elongación) y $72^{\circ} \mathrm{C}$ por $3 \mathrm{~min}$ (elongación final) (24). Para el análisis del polimorfismo L858R del exón 21 se utilizaron los primers FW 5' - ATG AAC TAC TTG GAG GAC CGT C -3' y RV 5' - TGC CTC CTT CTG CAT GGT ATT C -3' (24). Los pasos de la PCR fueron $95^{\circ} \mathrm{C}$ por $12 \mathrm{~min}$ (denaturación inicial), 
seguido de 35 ciclos a $94^{\circ} \mathrm{C}$ por $30 \mathrm{seg}$ (denaturación), $61^{\circ} \mathrm{C}$ por $30 \mathrm{seg}$ (anillamiento), $72^{\circ} \mathrm{C}$ por $30 \mathrm{seg}$ (elongación) y $72^{\circ} \mathrm{C}$ por $20 \mathrm{~min}$ (elongación final). Los productos de PCR fueron digeridos con la enzima de restricción Sau96I (corte cohesivo; G/GNCC) para la detección de la mutación L858R, y SacI (corte cohesivo; GAGCT/C) para la detección se G719S (24).

Análisis estadístico.- En este estudio retrospectivo casos-controles, la información de las características patológicas se recopiló en una base de datos y los análisis de chi-cuadrado $\left(\chi^{2}\right)$, odds ratio (OR) y su respectivo valor $p$, se calculó mediante el programa estadístico PASW 17 para Windows (SPSS, Chicago, IL). La prueba $\chi^{2}$ permitió determinar si existen diferencias significativas entre casos y controles con respecto a la presencia de las diferentes mutaciones y polimorfismos. La prueba OR permite determinar el riesgo que presentan los individuos con los polimorfismos estudiados con relación al desarrollo del cáncer. De igual forma se calcularon las frecuencias alélicas y genotípicas de cada uno de los genes estudiados en la población.

\section{RESULTADOS}

El promedio de edad de los 144 individuos afectos con cáncer de próstata fue de 70 años. El 27\% de los casos fue diagnosticado con cáncer avanzado. El $65 \%$ de los individuos afectos presentó niveles mayores de $10 \mathrm{ng} / \mathrm{mL}$ de antígeno prostático específico (PSA). La tabla 1 muestra la distribución genotípica y frecuencia alélica de los polimorfismos A49T y V89L del gen SRD5A2. La variante A49T presentó un $\chi^{2}$ de 27.29 ( $p$ $<0.001$ ), siendo altamente significativa entre individuos afectos y sanos; mientras que el OR fue $0.76(p=0.39)$. El genotipo TT estuvo presente en el $18 \%$ de los individuos afectos y no estuvo presente en los individuos sanos. Mientras que la variante V89L presentó un $\chi^{2}$ de $7.5(p<0.001)$ y un OR de $3.7(p<$ 0.001) (30).

Tabla 1. Distribución genotípica y frecuencia alélica de los polimorfismos A49T y V89L (SRD5A2) en cáncer de próstata.

\begin{tabular}{|c|c|c|c|c|c|c|c|}
\hline & & Casos & Controles & Frecu & cias alélicas & & \\
\hline Polimorfismos & Genotipo & $(n=114)$ & $(n=144)$ & Casos & Controles & Chi-cuadrado $\left(\chi^{2}\right)$ & Odds ratio (OR) \\
\hline \multirow[b]{2}{*}{ A49T } & $\mathrm{A} / \mathrm{A}$ & $33(29 \%)$ & $50(35 \%)$ & 0.55 & 0.67 & 27.29 & $0.76 ; 95$ IC $0.43-1.3$ \\
\hline & $\mathrm{A} / \mathrm{T}$ & $60(53 \%)$ & $94(65 \%)$ & & & \multirow{3}{*}{$\mathrm{p}<0.001$} & \multirow{3}{*}{$\mathrm{p}>0.05$} \\
\hline \multirow{6}{*}{ V89L } & & & & & & & \\
\hline & $\mathrm{T} / \mathrm{T}$ & $21(18 \%)$ & $0(0 \%)$ & 0.45 & 0.33 & & \\
\hline & $\mathrm{V} / \mathrm{V}$ & $46(40 \%)$ & $22(15 \%)$ & 0.68 & 0.47 & 7.5 & $3.7 ; 95 \%$ IC $2.1-6.7$ \\
\hline & & & & & & \multirow[t]{3}{*}{$\mathrm{p}<0.001$} & \multirow[t]{3}{*}{$\mathrm{p}<0.001$} \\
\hline & $\mathrm{V} / \mathrm{L}$ & $64(56 \%)$ & $91(63 \%)$ & & & & \\
\hline & $\mathrm{L} / \mathrm{L}$ & $4(4 \%)$ & $31(22 \%)$ & 0.32 & 0.53 & & \\
\hline
\end{tabular}

IC: Intervalo de confianza 
En cuanto a los 97 individuos afectos con cáncer de vejiga, el promedio de edad de los pacientes fue de 68 años. El $43 \%$ de ellos correspondió a mujeres y el $57 \%$ a hombres. El subtipo histológico de mayor porcentaje fue el carcinoma de células transicionales con un $86 \%$ en hombres y $93 \%$ en mujeres. La tabla 2 nos muestra la distribución genotípica y frecuencia alélica de los polimorfismos P198L y I58T de los genes GPX-1 y MnSOD, respectivamente. El análisis estadístico corroboró diferencia significativa y riesgo relativo con respecto a la presencia del polimorfismo P198L entre individuos afectos y sanos, obteniendo un $\chi^{2}$ de $69.9(p<0.001)$ y un riesgo OR de $3.8(p<0.001)$. Por el contrario, no se encontró relación entre el polimorfismo I58T y el desarrollo de cáncer de vejiga, obteniendo un $\chi^{2}$ de $0.25(p>0.05)$ y un riesgo OR no significativo de $2.1(p>$ $0.05)(20)$.
Sobre el análisis de 80 individuos afectos con cáncer de pulmón, se determinó que el promedio de edad de los pacientes con carcinoma pulmonar fue 68 años. El $65 \%$ de ellos fue hombre y el $35 \%$ fue mujer. El $69 \%$ de los pacientes fue fumador activo mientras que el $31 \%$ era fumador pasivo. La tabla 3 enlista la distribución genotípica y frecuencia alélica de las mutaciones L858R y G719S del oncogén EGFR. Diferencia altamente significativa y riesgo se encontró entre los individuos afectos con cáncer pulmonar y los individuos sanos con respecto a la mutación L858R, obteniendo un $\chi^{2}$ de $22.4(p<0.001)$ y un OR de $5.9(p<0.001)$. Mientras que, no se encontró diferencia significativa ni riesgo entre la presencia de la mutación G719S y el desarrollo de cáncer de pulmón, obteniendo un $\chi^{2}$ de $2.2(p>0.05)$ y un OR de $2.5(p>0.05)(24)$.

Tabla 2. Distribución genotípica y frecuencia alélica de los polimorfismos P198L (GPX-1) y I58T (MnSOD) en cáncer de vejiga.

\begin{tabular}{|c|c|c|c|c|c|c|c|}
\hline Polimorfismos & Genotipo & $\begin{array}{l}\text { Casos } \\
(\mathbf{n}=97)\end{array}$ & $\begin{array}{c}\text { Controles } \\
(\mathrm{n}=120)\end{array}$ & $\begin{array}{l}\text { Frecu } \\
\text { Casos }\end{array}$ & $\begin{array}{r}\text { cias alélicas } \\
\text { Controles }\end{array}$ & Chi-cuadrado $\left(\chi_{2}\right)$ & Odds ratio (OR) \\
\hline & $\mathrm{P} / \mathrm{P}$ & $28(29 \%)$ & $73(61 \%)$ & 0.39 & 0.79 & 69.9 & $3.8 ; 95 \%$ IC $2.1-6.8$ \\
\hline & $\mathrm{P} / \mathrm{L}$ & $19(19 \%)$ & $42(35 \%)$ & & & $\mathrm{p}<0.001$ & $p>0.001$ \\
\hline & $\mathrm{L} / \mathrm{L}$ & $50(52 \%)$ & $5(4 \%)$ & 0.61 & 0.21 & & \\
\hline & $\mathrm{I} / \mathrm{I}$ & $43(44 \%)$ & $75(62 \%)$ & 0.68 & 0.82 & 0.25 & 2,$1 ; 95 \%$ IC $1.3-3.5$ \\
\hline \multicolumn{8}{|l|}{$158 \mathrm{~T}$} \\
\hline & $\mathrm{I} / \mathrm{T}$ & $47(49 \%)$ & $45(38 \%)$ & & & & \\
\hline & $\mathrm{T} / \mathrm{T}$ & $7(7 \%)$ & $0(0 \%)$ & 0.32 & 0.18 & & \\
\hline
\end{tabular}

IC: Intervalo de confianza 
Tabla 3. Distribución genotípica y frecuencia alélica de las mutaciones L858R y G719S (EGFR) en cáncer de pulmón.

\begin{tabular}{|c|c|c|c|c|c|c|c|}
\hline \multirow{2}{*}{ Polimorfismos } & \multirow{2}{*}{ Genotipo } & \multirow{2}{*}{$\begin{array}{c}\text { Casos } \\
(\mathrm{n}=80)\end{array}$} & \multirow{2}{*}{$\begin{array}{c}\text { Controles } \\
(\mathrm{n}=109)\end{array}$} & \multicolumn{2}{|c|}{ Frecuencias alélicas } & \multirow{2}{*}{ Chi-cuadrado $\left(\chi_{2}\right)$} & \multirow{2}{*}{ Odds ratio (OR) } \\
\hline & & & & Casos & Controles & & \\
\hline \multirow{4}{*}{ L858R } & $\mathrm{L} / \mathrm{L}$ & $50(63 \%)$ & $99(91 \%)$ & 0.66 & 0.91 & 22.4 & $5.9 ; 95 \%$ IC $2.7-13.1$ \\
\hline & $L / R$ & $5(6 \%)$ & $1(1 \%)$ & & & $\mathrm{p}<0.001$ & $p<0.001$ \\
\hline & $\mathrm{R} / \mathrm{R}$ & $25(31 \%)$ & $9(8 \%)$ & 0.34 & 0.09 & & \\
\hline & $\mathrm{G} / \mathrm{G}$ & $73(91 \%)$ & $105(96 \%)$ & 0.92 & 0.96 & 2.2 & $2.5 ; 95 \%$ IC $0.71-8.9$ \\
\hline \multirow[t]{2}{*}{ G719S } & $\mathrm{G} / \mathrm{S}$ & $2(3 \%)$ & $1(1 \%)$ & & & & \\
\hline & $\mathrm{S} / \mathrm{S}$ & 5 & $3(3 \%)$ & 0.08 & 0.04 & $\mathrm{p}>0.05$ & $\mathrm{p}>0.05$ \\
\hline
\end{tabular}

IC: Intervalo de confianza

\section{DISCUSIÓN Y CONCLUSIONES}

La caracterización genética de la población ecuatoriana ha sido un pilar en el desarrollo de la investigación en cáncer en el Ecuador y en el entendimiento de la función de los genes y su asociación con las diferentes enfermedades. Se han estudiado los genes GPX-1 y Apo E en enfermedad de Alzheimer (14); CYP $1 \mathrm{~A} 1$ en individuos expuestos a genotóxicos $(4,5)$; CCR5 332, CCR2-641 y SDF1-3'A en individuos con resistencia a VIH-1 (31), $\Delta$ F508 en fibrosis quística (32); BCR/ABL en leucemia mieloide crónica y leucemia linfoide aguda (33); RB1 en retinoblastoma (34); hMSH2 en linfoma no Hodgkin (35); y NF2 en neurofibromatosis (36).

El cáncer es una de las principales causas de muerte a nivel mundial y debido a la exposición cada vez más frecuente a factores de riesgo como el tabaquismo, los rayos UV, los malos hábitos alimenticios, el estrés oxidativo, la edad, la exposición a carcinógenos como rayos $\mathrm{X}$, pesticidas e hidrocarburos, es de gran importancia entender el comportamiento de esta enfermedad a nivel celular y genético $(4,5,6)$.

Con respecto al gen SRD5A2 en cáncer de próstata, se determinó que el polimorfismos V89L presentó una diferencia estadísticamente significativa en la población afecta comparada con la población control $\left(\chi^{2}=7.5, p<\right.$ 0.001 ; OR $=3.7,95 \%$ Intervalo de confianza (IC) $2.1-6.7, p<0.001$ ), siendo un factor de riesgo para el desarrollo del carcinoma prostático (30). Por el contrario, otros estudios han reportado asociación negativa entre el polimorfismo V89L y este cáncer, sugiriendo que diversas poblaciones a nivel mundial presentan bajo riesgo de adquirir cáncer de próstata debido a una reducida actividad del esteroide $5 \alpha$-reductasa $(37,38,39)$. 
Con relación al cáncer de vejiga, el polimorfismo I58T del gen MnSOD no presentó asociación estadísticamente significativa $\left(\chi^{2}=0.25, p>0.05 ; \mathrm{OR}=\right.$ $2.1,95 \%$ IC $1.3-3.5, p>0.05$ ), mientras que el polimorfismo P198L del gen GPX-1 presentó diferencia estadísticamente significativa en población afecta $\left(\chi^{2}=69.9, p<0.001 ;\right.$ OR $=3.8,95 \%$ IC $2.1-6.8, p<0.001)$, demostrando que la presencia de la variante alélica $\mathrm{L} / \mathrm{L}$ deteriora la actividad enzimática de glutatión peroxidasa, permitiendo la acumulación de especies oxígeno reactivas en el organismo y, por lo tanto, confiriendo riesgo de desarrollar cáncer de vejiga. Individuos con la presencia de esta variante tienen cuatro veces mayor riesgo de adquirir esta enfermedad que los individuos con la variante alélica $\mathrm{P} / \mathrm{P}$, tal como lo demuestran estudios en población japonesa y caucásica (18, 40, 41).

Sobre las mutaciones L858R y G719S del cáncer pulmonar, se determinó que la variante L858R del dominio tirosina quinasa presentó asociación estadísticamente significativa con el cáncer de pulmón $\left(\chi^{2}=5.9, p<\right.$ $0.001 ;$ OR $=5.9,95 \%$ IC $2.7-13.1, p$ $<0.001)$. Estos resultados son corroborados por varios estudios internacionales que demuestran que las mutaciones EGFR se sobreexpresan entre el $40 \mathrm{y}$ $80 \%$ de individuos con cáncer pulmonar (42). En Japón, la frecuencia de las mutaciones EGFR varía entre el 25 y
40\% (43); en Estados Unidos entre el 10 y $30 \%$ (44); y en China alrededor del 18\% (45).

Es importante conocer las frecuencias de las mutaciones y polimorfismos de la población ecuatoriana afecta con diferentes tipos de cáncer, debido a que al determinar la predisposición genética para la sobreexpresión o inhibición de funciones de las proteínas se puede desarrollar tratamientos específicos para cada individuo a través del uso de inhibidores de tirosina quinasa o inhibidores de expresión génica mediante el uso de miARNs; se puede regular la proliferación celular y así controlar la formación de tumores; se puede activar los procesos de apoptosis para la muerte programada de células cancerígenas; se puede controlar los procesos de fosforilación intracelular para regular la diferenciación y proliferación celular; al igual que impedir la activación de procesos irregulares de angiogénesis para así ayudar a controlar la formación de células cancerígenas.

\section{AGRADECIMIENTOS}

La realización de las diferentes investigaciones en la genética, predisposición y desarrollo del cáncer, en la población ecuatoriana, han sido posibles gracias al apoyo de los Departamentos de Patología del Hospital Oncológico Solón Espinoza Ayala (SOLCA) y del Hospital Carlos Andrade Marín (HCAM). 


\section{REFERENCIAS}

\section{BIBLIOGRÁFICAS}

1. AMERICAN CANCER SOCIETY. 2010. Cancer Facts and Figures 2010. Página de Internet: www.cancer. org/acs/groups/content/@nho/ documents/document/acspc-024113. pdf. Consultada: 19 Mayo 2011.

2. MENDELSOHN, J.; HOWLEY, P.; ISRAEL, M.; LIOTTA, L. 1995. The molecular basis of cancer. W. B. Saunders Company. Philadelphia, USA.

3. VARMUS, H. \& WEINBERG, R. 1993. Genes and the biology of cancer. Scientific American Library. New York, USA.

4. PAZ-Y-MIÑO, C.; ARÉVALO, M.; SÁNCHEZ, M. E.; LEONE, P. 2004. Chromosome and DNA damage analysis in individuals occupationally exposed to pesticides with relation to genetic polymorphism for CYP1A1 gene in Ecuador. Mutation Research. 562: 77-89.

5. PAZ-Y-MIÑO, C.; LÓPEZCORTÉS, A.; ARÉVALO, M.; SÁNCHEZ, M. E. 2008. Monitoring of DNA damage in exposed individuals to petroleum hydrocarbons in Ecuador. Annals of the New York Academy of Sciences. 1140: 121-128.

6. MUÑOZ, M. J.; LÓPEZ-CORTÉS, A.; SARMIENTO, I.; HERRERA, C.; SÁNCHEZ, M. E.; PAZ-Y-
MIÑO, C. 2008. Biomonitoreo genético de individuos expuestos a radiación ionizante y su relación con el desarrollo de cáncer. Oncología, 18: $75-82$.

7. CUEVA, P. \& YÉPEZ, J. 2009. Epidemiología del cáncer en Quito 2003-2005. Registro Nacional de Tumores SOLCA. Quito, Ecuador.

8. POSTMA, R. \& SCHRÖDER, F. H. 2005. Screening for prostate cancer. European Journal of Cancer. 41: 825-833.

9. BOSLAND, M. 2006. Sex steroids and prostate carcinogenesis integrated, multifactorial working hypothesis. Annals of the New York Academy of Sciences. 1089: 168-176.

10. MAKRIDAKIS, N. M.; ROSS, R. K.; PIKE, M. C.; CHANG, L.; STANCZYK, F. Z.; KOLONEL, L. N.; SHI, C. Y.; YU, M. C.; HENDERSON, B.E.; REICHARDT, J. K. V. 1997. A prevalent missense substitution that modulates activity of prostatic steroids $5 \alpha$-reductase. Cancer Research. 57: 1020-1022.

11. ROSS, R. K.; PIKE, M. C.; COETZEE, G. A.; REICHARDT, J. K. V.; YU, M. C.; FEIGELSON, H.; STANCZYK, F. Z.; KOLONEL, L. N.; HENDERSON, B. E. 1998. Androgen metabolism and prostate cancer: Establishing a model of genetic susceptibility. Cancer Research. 58: 4497-4504. 
12. HSING, A. W.; CHEN, C.; CHOKKALINGAM, A. P.; GAO, Y. T.; DIGHTMAN, D. A.; NGUYEN, H. T.; DENG, J.; CHENG, J.; SESTERHENN, I. A.; MOSTOFI, F. K.; STANXZYK, F. Z.; REICHARDT, J. K. V. 2001. Polymorphic markers in the SRD5A2 gene and prostate cancer risk: a population-based case-control study. Cancer Epidemiology, Biomarkers \& Prevention. 57: 1020-1022.

13. SINGH, A.; CHAU, C. H.; PRICE, D. K.; FIGG, W. D. Mechanism of disease: polymorphisms of androgen regulatory genes in the development of prostate cancer. Nature Clinical Practice. 2: 101-107.

14. PAZ-Y-MIÑO, C.; CARRERA, C.; LÓPEZ-CORTÉS, A.; MUÑOZ, M. J.; HERRERA, C.; ROBLES, P.; CUMBAL, N.; SÁNCHEZ, M. E. 2009. Genetic polymorphisms in apolipoprotein $\mathrm{E}$ and glutathione peroxidase 1 genes in the Ecuadorian population affected with alzheimer disease. The American Journal of the Medical Sciences. 350(5): 373-377.

15. WU, X.; LIN, X.; DINNEY, C.; GU, J.; GROSSMAN, B. 2007. Genetic polymorphism in bladder cancer. Frontiers in Bioscience, 12: 192-213.

16. LEO PASBOS, C.; BOTTERMAN, M. F.; LASKIN, B. L.; REDAELLI, A. 2002. Bladder cancer: Epidemiology, diagnosis and management. Cancer Practice. 10(6): 311-322.
17. INSTITUTO NACIONAL DE ESTADÍSTICAS Y CENSOS. 2005. Anuario de estadísticas vitales: Nacimientos y defunciones. Quito, Ecuador.

18. ICHIMURA, Y.; HABUCHI, T.; TSUCHIYA, N. 2004. Increased risk of bladder cancer associated with a Glutathione peroxidase 1 codon 198 variant. Journal of Urology. 172: 728-732.

19. BORGSTAHL, G. E.; PARGE, H. E.; HICKEY, M. J. 1996. Human mitochondrial manganese superoxidase dismutase polymorphic variant Ile59Thr reduces activity by destabilizing the tetrameric interface. Biochemistry. 35: 4287-4297.

20. PAZ-Y-MIÑO, C.; MUÑOZ, M. J.; LÓPEZ-CORTÉS, A.; CABRERA, A.; PALACIOS, A.; CASTRO, B.; PAZ-Y-MIÑO, N.; SÁNCHEZ, M. E. 2010. Frequency of polymorphisms pro198leu in GPX-1 gene and ile58thr in MnSOD gene in the altitude Ecuadorian population with bladder cancer. Oncology Research. 18: 395-400.

21. ULLRICH, A.; COUSSEN, L.; HAYFLICK, J. S.; DULL, T. J.; GRAY, A.; TAM, A. W.; LEE, J.; YARDEN, Y.; LIBERMANN, T. A.; SCHLESSINGER, J.; DOWNWARD, J.; MAYES, E.; WHITTLE, N.; WATERFIELD, M. D.; SEEBURG, P. H. 1984. Human epidermal growth factor receptor 
cDNA sequence and aberrant expression of the amplified gene in A431 epidermoid carcinoma cells. Nature. 309: 418-425.

22. SOLTOFF, S. 1998. Related adhesion focal tyrosine kinase and the epidermal growth factor receptor mediate the stimulation of mitogenactivated protein kinase by the $G$ protein-coupled $\mathrm{P} 2 \mathrm{Y} 2$ receptor: phorbol ester or $\mathrm{Ca}^{2+}$ elevation can substitute for receptor activation. The Journal of Biological Chemistry. 273: 23110-23107.

23. JORRISSEN, R.; WALKER, F.; POULIOT, N. 2003. Epidermal growth factor receptor: mechanisms of activation and signaling. Experimental Cell Research. 284: 31-35.

24. PAZ-Y-MIÑO, C.; LÓPEZCORTÉS, A.; MUÑOZ, M. J.; CABRERA, A.; CASTRO, B.; SÁNCHEZ, M. E. 2010. Incidence of the L858R and G719S mutations of the epidermal growth factor receptor oncogene in an Ecuadorian population with lung cancer. Cancer Genetics \& Cytogenetics. 196: 201203.

25. BARTLETT, J. \& STIRLING, D. 2003. A Short History of the Polymerase Chain Reaction. Methods in Molecular Biology. 226: 3-6

26. SUDBERY, P. 2004. Genética Molecular Humana. Segunda edición. Pearson Prentice Hall. Madrid, España.
27. STRACHAN, T. \& READ, A. 1999. Human Molecular Genetics 2. Second edition. BIOS Scientific Publishers Ltd. Oxford, UK.

28. PAN, Q.; PAO, W.; LADANYI, M. 2005. Rapid polymerase chain reaction-based detection of epidermal growth factor receptor gene mutations in lung adenocarcinomas. Journal of Molecular Diagnostics. 7(3): 396-403.

29. RIELY, G.; PAO, W.; PHAM, D.; LI, A.; RIZVI, N.; VENKATRAMAN, E.; ZAKOWSKI, M.; KRIS, M.; LADANYI, M.; MILLER, V. 2006. Clinical course of patients with nonsmall cell lung cancer and epidermal growth factor receptor exon 19 and exon 21 mutations treated with Gefitinib or Erlotinib. Clinical Cancer Research. 12(3): 839-844.

30. PAZ-Y-MIÑO, C.; WITTE, T.; ROBLES, P.; LLUMIPANTA, W.; DÍAZ, M.; ARÉVALO, M. 2009. Association among polymorphisms in the steroid $5 \alpha$-reductase type II (SRD5A2) gene, prostate cancer risk, and pathologic characteristics of prostate tumors in an Ecuadorian population. Cancer Genetics \& Cytogenetics. 189: 71-76.

31. PAZ-Y-MIÑO, C.; MURILLO, S.; CELI, A.; WITTE, T.; MUÑOZ, M. J.; COLLANTES, J.C.; LEONE, P. 2005. CCR5 $\triangle 32$, CCR2-641 and SDF1-3'A polymorphisms related to resistance to HIV-1 infection and 
disease in the Ecuadorian population. Human Biology. 77(4): 521-526.

32. PAZ-Y-MIÑO, C.; PÉREZ, J. C.; BURGOS, R.; DÁVALOS, M. V.; LEONE, P. 1999. The $\triangle F 508$ mutation in Ecuador, South America. Human Mutation. 14: 348-350.

33. PAZ-Y-MIÑO, C.; BURGOS, R.; MORILLO, S.; SANTOS, J. C.; FIALLO, F.; LEONE, P. 1998. BCR$\mathrm{ABL}$ rearrangement frequencies in CML and ALL in Ecuador, South America. Cancer Genetics and Cytogenetics. 132: 65-67.

34. LEONE, P.; VEGA, M. E.; JERVIS, P.; PESTAÑA, A.; ALONSO, J.; PAZ-Y-MIÑO, C. 2003. Two new mutations and three novel polymorphisms in the RB1 in Ecuadorian patients. Journal of Human Genetics. 1-5.

35. PAZ-Y-MIÑO, C.; PÉREZ, J. C.; BURGOS, R.; LEONE, P. 2001. A polymorphism in the hMSH2 gene (Givs12-6T $>$ C) associated with non-Hodking lymphomas. Cancer Genetics \& Cytogenetics. 133: 2933.

36. PAZ-Y-MIÑO, C. \& LEONE, P. 2000. Three novel somatic mutations in the NF2 tumor supresor gene (g816T $>$ A; g1159A $>$ G; gIVS11$1 \mathrm{G}>\mathrm{T})$. Human Mutation. 15(5): 487.

37. CICEK, M.; CONTI, D.; CURRAN, A.; NEVILLE, P.; PARIS, P.; CASEY, G.; WITTE, J. 2004.
Association of prostate cancer risk and aggressiveness to androgen pathway genes: SRD5A2, CYP17, and the AR. Prostate. 59: 69-76.

38. HAYES, V.; SEVERI, G.; PADILLA, E.; MORRIS, H., TILLEY, W.; SOUTHEY, M.; ENGLISH, D.; SUTHERLAND, R.; HOPPER, J.; BOYLE, P.; GILES, G. 2006. $5 \alpha$-reductase type II gene variant association with prostate cancer risk, circulating hormone levels and androgenic alopecia. International Journal of Cancer. 120: 776-780.

39. SHIBATA, C.; GARCÍA, M.; CHENG, I.; STAMEY, T.; McNEAL, J.; BROOKS, J.; HENDERSON, S.; YEMOTO, C.; PEELH, D. 2002. Polymorphisms in the androgen receptor and type II $5 \alpha$-reductase genes and prostate cancer prognosis. Prostate. 52: 269-278.

40. RATNASINGHE, D.; TANGREA, J.; ANDERSEN, M. 2000. Glutathione peroxidasa codon 198 polymorphism variant increases lung cancer risk. Cancer Research. 60: 6381-6383.

41. HU, Y; DIAMOND, A. 2003. Role of glutathione peroxidasa 1 in breast cancer. Loss of heterozygosity and allelic differences in the response to selenium. Cancer Research. 63: 3347.

42. YEH, K.; YEH, S.; WAN, J.; SHEN, Y.; CHENG, A. 2005. Somatic mutations in epidermal 
growth factor receptor underlying complete responsiveness to gefitinib in a Taiwanese female patient with metastatic adenocarcinoma of lung. Anticancer drugs. 16(7): 739-742.

43. PAEZ, J.; JANNE, P.; LEE, J.; TRACY, S.; GREULICH, H.; GABRIEL, S.; HERMAN, P.; KAYE, F.; LINDEMAN, N.; BOGGON, T.; NAOKI, K.; SASAKI, H.; FUJII, Y.; ECK, M.; SELLERS, W.; JOHNSON, B.; MEYERSON, M. 2004. EGFR mutations in lung cancer: correlation with clinical response to gefitinib therapy. Science. 304: 1497-1500.
44. ZHAO, Y.; LI, Q.; LI, X.; HAN, W.; HAO, H.; WU, Z. 2007. Epidermal growth factor receptor mutation in non-small cell lung cancer and breast cancer. Yi chuan. 29(5):547-553.

45. HAN, S.; KIM, T.; HWANG, P. 2005. Predictive and prognostic impact of epidermal growth factor receptor mutation in non smallcell lung cancer patients treated with gefitinib. Journal of Clinical Oncology. 23:2493-2501. 